\title{
Language mapping in children using resting-state functional connectivity: comparison with a task-based approach
}

\author{
Anne Gallagher \\ Julie Tremblay \\ Phetsamone Vannasing
}




\title{
Language mapping in children using resting-state functional connectivity: comparison with a task-based approach
}

\author{
Anne Gallagher, ${ }^{a, b, *}$ Julie Tremblay, ${ }^{a, b}$ and Phetsamone Vannasing ${ }^{a, b}$ \\ ${ }^{a} \mathrm{CHU}$ Sainte-Justine Research Center, Laboratoire d'Imagerie Optique en Neurodéveloppement (LIONLab), \\ 3175 Chemin de la Côte-Sainte-Catherine, Montréal, Québec H3T 1C5, Canada \\ bUniversité de Montréal, Centre de Recherche en Neuropsychologie et Cognition, Department of Psychology, Marie-Victorin Building, \\ P.O. Box 6128 Centre-ville Station, 2900 Boulevard Édouard-Montpetit, Montréal, Québec H3T 1J4, Canada
}

\begin{abstract}
Patients with brain tumor or refractory epilepsy may be candidates for neurosurgery. Presurgical evaluation often includes language investigation to prevent or reduce the risk of postsurgical language deficits. Current techniques involve significant limitations with pediatric populations. Recently, near-infrared spectroscopy (NIRS) has been shown to be a valuable neuroimaging technique for language localization in children. However, it typically requires the child to perform a task (task-based NIRS), which may constitute a significant limitation. Resting-state functional connectivity NIRS (fcNIRS) is an approach that can be used to identify language networks at rest. This study aims to assess the utility of fcNIRS in children by comparing fcNIRS to more conventional task-based NIRS for language mapping in 33 healthy participants: 25 children (ages 3 to 16) and 8 adults. Data were acquired at rest and during a language task. Results show very good concordance between both approaches for language localization (Dice similarity coefficient $=0.81 \pm 0.13$ ) and hemispheric language dominance (kappa $=0.86, p<0.006$ ). The fcNIRS technique may be a valuable tool for language mapping in clinical populations, including children and patients with cognitive and behavioral impairments. () The Authors. Published by SPIE under a Creative Commons Attribution 3.0 Unported License. Distribution or reproduction of this work in whole or in part requires full attribution of the original publication, including its DOI. [DOI: 10.1117/1.JBO.21.12.125006]
\end{abstract}

Keywords: language network; presurgical mapping; resting-state functional connectivity; pediatric populations; task-based near-infrared spectroscopy; functional connectivity near-infrared spectroscopy.

Paper 160517R received Jul. 26, 2016; accepted for publication Nov. 21, 2016; published online Dec. 19, 2016.

\section{Introduction}

Brain localization and lateralization of language functions are of great importance in the presurgical assessment of patients with intractable epilepsy and brain tumor. Presurgical investigation for language functions usually requires electrical stimulation mapping (ESM), an intracarotid amobarbital test (IAT), also known as the Wada test, and functional neuroimaging. ${ }^{1}$ Unfortunately, few of these techniques are suitable for children due to their significant drawbacks. For instance, ESM and IAT are highly invasive procedures that carry the risk of severe complications, including stroke, hemorrhage, and infection $(0.5 \%$ to $5 \%$ for $\mathrm{ESM}^{2}$ and $1 \%$ to $11 \%$ for $\mathrm{IAT}^{3,4}$ ). Furthermore, these invasive procedures require the patient to remain alert for language assessment, which is unfeasible in half of children with intractable epilepsy due to cognitive and behavioral problems. ${ }^{1,5}$ Consequently, inconclusive results are reported in most patients younger than age 10 for ESM (up to 81\%) and for IAT (50\%).,

In the past decade, noninvasive neuroimaging techniques, including functional magnetic resonance imaging (fMRI) ${ }^{7-11}$ and magnetoencephalography (MEG), ${ }^{12-18}$ have been widely used for language cerebral lateralization and mapping in adults and children. These techniques show very good concordance with invasive procedures (IAT and ESM) for language dominance and localization. ${ }^{19-23}$ However, as with ESM and IAT, these noninvasive techniques are unsuitable for young children and patients

*Address all correspondence to: Anne Gallagher, E-mail: anne.gallagher@ umontreal.ca with severe cognitive or behavioral problems because their confined environment often elicits anxiety in these patients. In addition, fMRI and MEG are known to be highly sensitive to motion artifacts related to head movements or to muscle and articulatory movements in language production aloud, which is crucial in these populations to control for task performance.

Alternatively, near-infrared spectroscopy (NIRS) has also been used for language localization and lateralization in healthy and clinical populations. Analogous to fMRI, it measures changes in cerebral blood volume and hemoglobin concentrations, including oxy-hemoglobin $(\mathrm{HbO})$ and deoxy-hemoglobin $(\mathrm{HbR})$, which are thought to be associated with neural activity. ${ }^{24}$ Multiple studies ${ }^{25-27}$ have shown strong correlations between NIRS data and fMRI, Single photon emission computed tomography, IAT, and ESM results in patients with epilepsy, confirming the usefulness of NIRS for presurgical mapping. NIRS is more suitable for young children and challenged populations ${ }^{28,29}$ than other neuroimaging techniques due to its tolerance to movement. Specifically, optical fibers are mounted on a helmet placed on the patient's head. Hence, regardless of head motion, the recording sites remain unchanged. In addition, data can be acquired while the child is performing a language task aloud, allowing task performance to be measured. Another major advantage is that the parent can stay with the child during data acquisition, which may be very reassuring for young children or patients with anxiety issues. Finally, NIRS is easily accessible since it is portable and relatively low in cost.

A number of epilepsy clinics around the world now use fMRI, MEG, and NIRS, which, in some patients, reduce the need for 
invasive investigation or the extent of the brain area studied using intracranial mapping. Although these noninvasive neuroimaging techniques have many advantages, they require the patient to perform a task during recording (for instance, a verbal fluency task for identifying language areas), which can take up to 45 min of data recording and is difficult to perform for young children and individuals with moderate or severe cognitive and behavioral impairments. As a result, these methods are often not available to these patients, which may rule out surgical treatment or increase the risk of postoperative deficits. ${ }^{5,30}$

Resting-state functional connectivity is an alternative neuroimaging approach that is not subject to the limitations described above. It is a relatively a modern method for evaluating the regional interactions that occur in the brain when a subject is not performing an explicit task. With this approach, specific cognitive networks in the brain, such as the language network, can be identified without stimulation or active subject participation by localizing temporally correlated brain signals at rest. ${ }^{31}$ This makes the technique ideal for use with children and cognitively challenged populations, as it does not require the participant to perform a task or follow instructions during data acquisition. Although it can be applied to most neuroimaging techniques, it was first demonstrated in fMRI, where temporal correlations in spontaneous blood oxygen level-dependent signal oscillations were detected between regions while subjects were resting quietly in the scanner. ${ }^{32}$ Temporally correlated signal fluctuations are presumed to reflect intrinsic functional connectivity. By recording the participant at rest for several minutes, it is thus possible to study and identify various functional networks, including language (see Ref. 33 for review).

As with fMRI, studies have confirmed the feasibility of applying resting-state functional connectivity to NIRS (fcNIRS) in order to investigate language networks in healthy adults and children at rest, without performing a task. ${ }^{34-37}$ Brain mapping with fcMRI and fcNIRS has shown good spatial correlations, ${ }^{38,39}$ and good test-retest reliability has been reported with fcNIRS. ${ }^{40}$ The higher temporal resolution of NIRS ( $\sim 1 \mathrm{~ms})$ as compared to fMRI (seconds ${ }^{41}$ may prevent confounding of physiological noise, such as respiratory and cardiovascular activity, with intrinsic activity. ${ }^{42-44}$

The goal of this study is to assess the utility of fcNIRS for language brain mapping in children by comparing language network maps at rest, using fcNIRS, and while participants perform a language task, using task-based NIRS. As such, we present a comparison of fcNIRS and task-based NIRS approaches for language mapping in children.

\section{Materials and Methods}

\subsection{Participants}

In total, 33 participants were recruited for the study. Twenty-five healthy French-speaking children, ages 3 to 16, were recruited and assigned to an age-specific group. In addition, a group of eight young adults (ages 18 to 30) was included to measure mature language networks. Groups were defined based on previous studies,${ }^{45,46}$ allowing for the investigation of fcNIRS applicability at various developmental stages: early childhood (ages 3 to $6 ; n=6$ ), late childhood (ages 7 to $10 ; n=8$ ), adolescence (ages 11 to $16 ; n=11$ ), and early adulthood (ages 18 to 30 ; $n=8$ ). Exclusion criteria were preterm birth (gestational age $<37$ weeks) or history of known congenital, neurologic, developmental, psychiatric, or metabolic disorders. Demographic data for participants are presented in Table 1. This project was approved
Table 1 Demographic information.

\begin{tabular}{|c|c|c|c|c|}
\hline Participant & Group & Age (yr) & Gender & Handedness \\
\hline 1 & Early childhood & 3 & $\mathrm{~F}$ & $\mathrm{R}$ \\
\hline 2 & Early childhood & 4 & M & $\mathrm{R}$ \\
\hline 3 & Early childhood & 4 & $\mathrm{~F}$ & $\mathrm{R}$ \\
\hline 4 & Early childhood & 5 & M & $\mathrm{R}$ \\
\hline 5 & Early childhood & 6 & $\mathrm{~F}$ & $\mathrm{R}$ \\
\hline 6 & Early childhood & 6 & $\mathrm{~F}$ & B \\
\hline 7 & Late childhood & 7 & M & B \\
\hline 8 & Late childhood & 8 & $\mathrm{~F}$ & $\mathrm{R}$ \\
\hline 9 & Late childhood & 8 & $\mathrm{~F}$ & $\mathrm{R}$ \\
\hline 10 & Late childhood & 8 & $M$ & $\mathrm{R}$ \\
\hline 11 & Late childhood & 9 & $\mathrm{~F}$ & $\mathrm{R}$ \\
\hline 12 & Late childhood & 10 & $M$ & $\mathrm{R}$ \\
\hline 13 & Late childhood & 10 & M & $\mathrm{R}$ \\
\hline 14 & Late childhood & 10 & $\mathrm{~F}$ & $\mathrm{R}$ \\
\hline 15 & Adolescence & 11 & $\mathrm{~F}$ & $\mathrm{R}$ \\
\hline 16 & Adolescence & 13 & M & B \\
\hline 17 & Adolescence & 13 & $\mathrm{~F}$ & $\mathrm{R}$ \\
\hline 18 & Adolescence & 14 & $M$ & $\mathrm{R}$ \\
\hline 19 & Adolescence & 14 & $\mathrm{~F}$ & $\mathrm{R}$ \\
\hline 20 & Adolescence & 15 & $M$ & $\mathrm{R}$ \\
\hline 21 & Adolescence & 15 & M & $\mathrm{R}$ \\
\hline 22 & Adolescence & 15 & $M$ & $\mathrm{R}$ \\
\hline 23 & Adolescence & 15 & $\mathrm{~F}$ & $\mathrm{R}$ \\
\hline 24 & Adolescence & 16 & M & $\mathrm{R}$ \\
\hline 25 & Adolescence & 16 & $\mathrm{~F}$ & $\mathrm{R}$ \\
\hline 26 & Adulthood & 20 & $M$ & B \\
\hline 27 & Adulthood & 23 & $\mathrm{~F}$ & $\mathrm{R}$ \\
\hline 28 & Adulthood & 23 & M & $\mathrm{R}$ \\
\hline 29 & Adulthood & 23 & $\mathrm{~F}$ & $\mathrm{R}$ \\
\hline 30 & Adulthood & 26 & $\mathrm{~F}$ & $\mathrm{R}$ \\
\hline 31 & Adulthood & 28 & M & $\mathrm{R}$ \\
\hline 32 & Adulthood & 25 & $\mathrm{~F}$ & $\mathrm{R}$ \\
\hline 33 & Adulthood & 30 & $M$ & $\mathrm{R}$ \\
\hline
\end{tabular}


by the Institutional Ethics Committees, and written informed consent was obtained from all participants or their parents (for children and adolescents under 14 years).

\subsection{Near-Infrared Spectroscopy Data Acquisition and Procedure}

NIRS recording parameters and fibers montage were the same for fcNIRS and task-based NIRS. The only methodological differences between the two approaches were the duration of the data acquisition periods and, most importantly, further data analyses (see details below).

To compare fcNIRS and task-based NIRS language mapping, NIRS data were acquired at rest and during an expressive language task in a single recording session. For both fcNIRS and task-based NIRS, data acquisition was performed using a frequency-domain device (Imagent, ISS Inc., Illinois) equipped with 8 detectors and 64 sources (32 at 690-nm wavelength and 32 at $830 \mathrm{~nm}$ ). Optical intensity (DC), modulation amplitude (AC), and phase data were obtained at an acquisition rate of $19.5312 \mathrm{~Hz}$. Light sources and detectors were held in place using a comfortable, age-adapted helmet. Source-detector distances were set between 2 and $5 \mathrm{~cm}$, optimizing recording of the brain signal from various depths in the regions of interest. A standard montage was created and adapted for each age group according to the 10-20 system and the corresponding Brodmann areas (BA). NIRS fibers covered bilateral frontal and temporal areas so as to record brain activity in anterior and posterior languagerelated regions, Broca's (BA 44 and 45) and Wernicke's (BA 22, 39, and 40) areas, respectively [Fig. 1(a)], and their right hemisphere counterparts. For each participant, the accurate localization of each source and detector, as well as four fiducial points (nasion, left and right preauriculars, and tip of the nose), was digitized and recorded with the stereotaxic system Brainsight ${ }^{\mathrm{TM}}$ Frameless 39 (Rogue Research Inc., Montreal, Quebec, Canada) allowing for individual registration and reconstitution of the montage on an MRI template (Colin27, see Ref. 47).
NIRS data were acquired at rest (fcNIRS) and during a language task (task-based NIRS) in all participants, with periods of rest and language task acquired alternatively. A total of at least 12 blocks, including rest and language task, were recorded. Each block lasted $90 \mathrm{~s}$, which included a 30-s rest period followed by $30 \mathrm{~s}$ of language task and another 30 -s rest, allowing the hemodynamic response to return to the baseline before the next block (see Fig. 2 for block timeline).

For fcNIRS, the last $25 \mathrm{~s}$ of data were extracted from the first 30 -s rest period of each block (see * in Fig. 2). The first 5 s were excluded as they often include trigger artifacts $(2 \mathrm{~s}$ of machine drift). A total of 5 min ( 12 blocks $\times 25 \mathrm{~s})$ of fcNIRS data was recorded while participants were resting with eyes open to prevent drowsiness. The participants' alertness was monitored through an infrared camera during the recording session. During rest periods, participants were instructed not to think or rehearse the items produced in the previous task-based block, but to relax, stay quiet, and gaze down at a fixation cross on the computer screen. In addition to the last rest period (60 to 90 s of each 90 -s block), which allowed residual activation from the language task to return to the baseline, a break of 2 to 3 min between blocks was introduced to clear participants' minds from rehearsing the previous category noun.

For task-based NIRS, the expressive language task was a categorical verbal fluency one (in a given time, saying as many words as possible within a given category, such as colors, toys, animals, and so on) that was used in many of our previous studies. ${ }^{25,46,48-50}$ It was performed overtly to ensure that the participant was doing the task correctly. Using the Presentation software (Neurobehavioral Systems Inc., California), semantic categories were successively and visually presented on a monitor in a block-design paradigm. All participants were given practice sessions before the recording; in addition, nonreader children received an auditory assistance for the category nouns at the outset of each stimulus block. All participants completed at least 12 periods of language task, each associated with a different semantic category meaningful to young children.

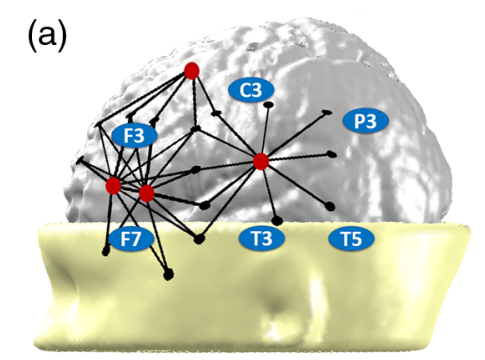

(d)

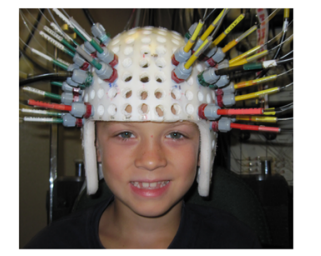

(b)

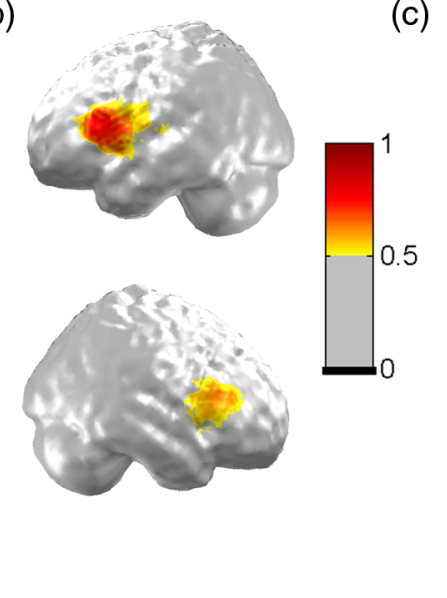

(c)

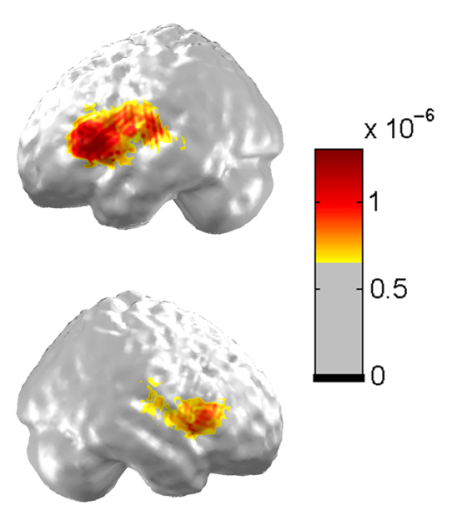

Fig. 1 Language mapping in all participants $(n=33)$ using fcNIRS and task-based NIRS. (a) Schematic view of the brain regions covered by the montage. Source (small dots, combined 690 and $830 \mathrm{~nm}$ ) and detector (red big dots) locations over bilateral frontal and temporal areas (only left hemisphere is shown) are projected on an MRI-template three-dimensional reconstruction. (b) Averaged fcNIRS language mapping in the left (top) and right (bottom) hemispheres reveals a left-hemispheric dominance for language networks. (c) Averaged language cerebral response during a verbal fluency task using task-based NIRS. (d) A picture of a participant wearing a helmet including probes setup. Similarly to fcNIRS findings, results show stronger brain activation in the left (top) compared to the right (bottom) hemisphere using this approach. 


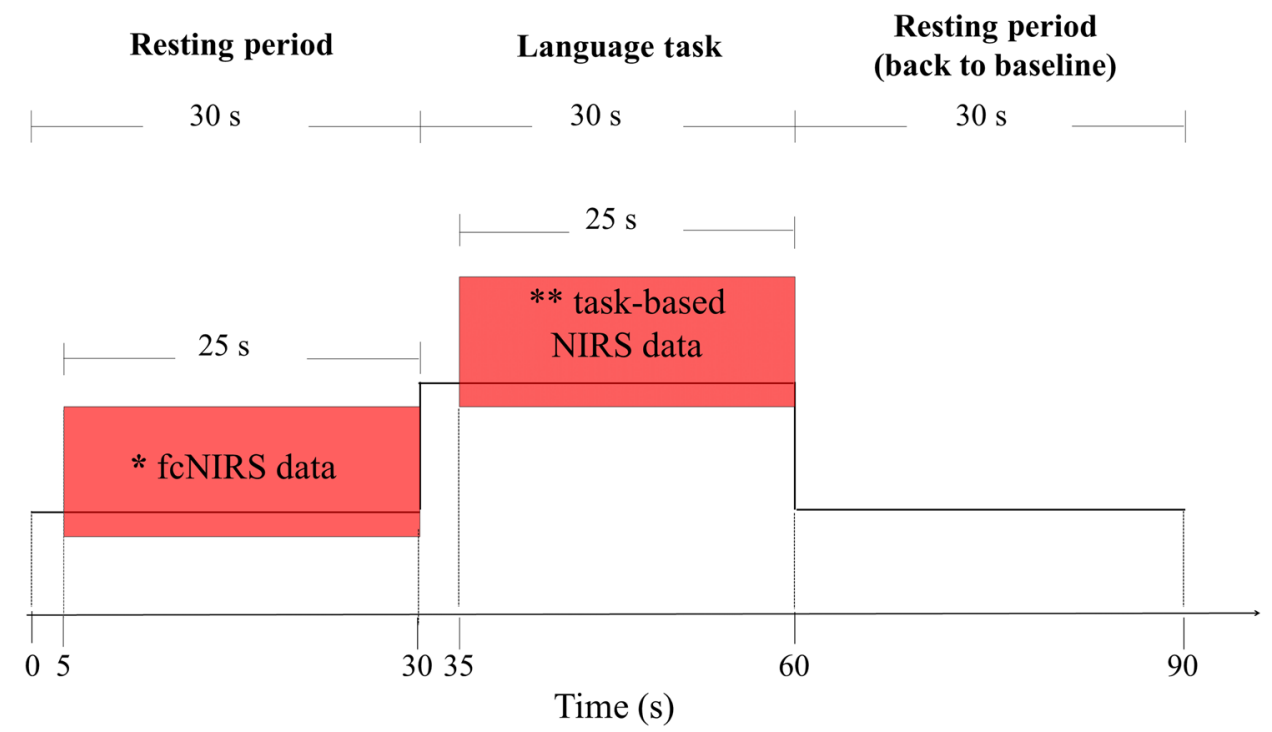

Fig. 2 Block timeline. Each NIRS recording block included periods of rest and language task allowing for computation of fcNIRS and task-based NIRS analyses. Each block lasted $90 \mathrm{~s}$, including a 30 -s rest period, followed by $30 \mathrm{~s}$ of language task, and another 30 -s rest. fcNIRS data were 25-s periods extracted from the first 30-s rest period of each block (see *). Task-based NIRS data were 25-s periods extracted from 30 -s language task periods (see ${ }^{\star \star}$ ).

NIRS data that were recorded between 5 and $30 \mathrm{~s}$ of the 30 -s language task periods were used for further task-based NIRS analyses (see ** in Fig. 2). Exclusion of the first $5 \mathrm{~s}$ after stimuli onset ensures that $\mathrm{HbO}$ and $\mathrm{HbR}$ changes reflect the participant's verbal production and not the auditory effect from the auditory assistance in nonreader children.

During the testing session, the participant was seated in a comfortable chair, placed $\sim 45$ in. from a 20 -in. computer screen. For children, parents were permitted to stay in the recording room if necessary. Prior to data acquisition, manual dominance was assessed using the Edinburgh inventory ${ }^{51}$ and a child-friendly homemade version of the Edinburgh inventory for participants younger than 10 years.

\subsection{Near-Infrared Spectroscopy Data Analyses}

A homemade graphical user interface program designed with MATLAB $^{\circledR}$ (The MathWorks, Inc., Massachusetts) was used to analyze fcNIRS and task-based NIRS data. Optical intensity (DC) data were normalized by dividing each value by the mean value across time points for each block and channel. Artifact rejection $( \pm 10 \%$ variation from normalized intensity) was applied to remove motion artifacts. Artifact-free data were then filtered for the effects of respiratory and cardiac signal with a cutoff frequency of $0.2 \mathrm{~Hz}$. HbO concentrations were calculated for each channel using the modified Beer-Lambert law with a differential path length adjusted to participants' age. ${ }^{52,53}$ $\mathrm{HbO}$ data, which are highly correlated with cerebral blood flow and have a better signal-to-noise ratio than HbR data, were used for fcNIRS and task-based NIRS analyses and comparisons. ${ }^{54,55}$

\subsubsection{Specific functional connectivity near-infrared spectroscopy analysis}

A seed-based correlation approach was used for resting-state language network mapping. The "seed" was identified as the most central channel in Broca's area and its right counterpart region (left or right frontal inferior gyrus). Temporal correlations among $\mathrm{HbO}$ concentration changes were extracted from the seed, and all other channels were calculated for each block. Correlation matrices obtained from all 12 blocks were averaged. ${ }^{56}$ To obtain language functional connectivity maps, $\mathrm{HbO}$ correlation results were coregistered and projected on an age-matched MRI template. ${ }^{57,58}$

Individual hemispheric language dominance for resting-state functional connectivity data was also identified using laterality indices (rs-fc LIs). The rs-fc LIs were calculated from $\mathrm{HbO}$ data by comparing the correlation strengths of the two brain hemispheres using the seed placed either in the left (L) or right (R) frontal inferior gyrus. For each participant, a difference map was calculated on the correlation between the left hemisphere seed and the left hemisphere target regions (LL), the left hemisphere seed and the right hemisphere target regions (LR), and the right hemisphere seed with homologous (RR) and contralateral (RL) target regions. From these seed versus target correlations (LL, $\mathrm{RL}, \mathrm{RR}$, and RL), rs-fc LIs were then calculated using the following equation:

$$
\begin{aligned}
\mathrm{rs}-\mathrm{fc} \mathrm{LI}= & (\mathrm{LL}-\mathrm{RL})-(\mathrm{RR}-\mathrm{LR}) /|\mathrm{LL}|+|\mathrm{LR}| \\
& +|\mathrm{RR}|+|\mathrm{RL}|,
\end{aligned}
$$

where LL-RL is the left targeted region on the difference map and RR-LR is the right targeted region on the difference map (for detailed methodology, see Ref. 31). The values of rs-fc LIs range between -1 and +1 , where positive values $(0.10$ to 1) indicate left language lateralization and negative values $(-1$ to -0.10$)$ indicate right dominance. ${ }^{59}$ Values between -0.10 and 0.10 are considered bilateral language lateralization.

\subsubsection{Specific task-based near-infrared spectroscopy analysis}

Variations in $\mathrm{HbO}$ concentrations associated with the language task were averaged for each channel across blocks ( 5 to $25 \mathrm{~s}$ from the 30 -s language task periods). $\mathrm{HbO}$ values were 
coregistered and projected on an age-matched MRI template ${ }^{57,58}$ to create and visualize the functional language map. Task-based laterality indices (task-based LIs) were also derived from $\mathrm{HbO}$ data from the two hemispheres using the following equation:

task-based $\mathrm{LI}=(L-R) /(L+R)$,

where $L$ is the maximal increase in $\mathrm{HbO}$ of all averaged channels covering Broca's area during the expressive language task and $R$ is the maximal increase in $\mathrm{HbO}$ of all averaged channels covering the homologous right area. ${ }^{25}$ Similarly to rs-fc LIs, the values of task-based LIs range between -1 and +1 , where positive values ( 0.10 to 1$)$ indicate left language lateralization and negative values $(-1$ to -0.10$)$ indicate right dominance. ${ }^{59}$ Values between -0.10 and 0.10 are considered bilateral language lateralization.

\subsection{Functional Connectivity Near-Infrared Spectroscopy and Task-Based Near-Infrared Spectroscopy Statistical Comparisons}

The Dice similarity coefficient (DSC) was used to compare language mapping obtained with each fcNIRS and task-based NIRS approach. The DSC is a measure of overlap and similarity between two maps (range: 0 to 1 , where $0=$ no overlap and $1=$ complete overlap) ${ }^{60}$ Based on the literature, ${ }^{60,61}$ a DSC $\geq 0.80$ was deemed to reflect very good concordance between maps, a DSC $\geq 0.70$ was deemed to reflect good concordance between maps, and a DSC $<0.70$ was associated with poor concordance. A one-way ANOVA on DSC values was performed to compare fcNIRS and task-based NIRS language mapping concordance between groups.

Concordance between individual language lateralization results was computed from both fcNIRS and task-based NIRS approaches using kappa statistic, which consists of comparing the results of rs-fc LIs and task-based LIs. ${ }^{62}$

\section{Results}

All participants showed clear language brain networks with fcNIRS as well as cerebral response to the language task in language-related cerebral areas, including Broca's and Wernicke's areas, with the task-based NIRS approach. Group and individual language localization and lateralization results are described below.

\subsection{Language Localization}

Figure 1 shows averaged language maps from fcNIRS [Fig. 1(b)] and task-based NIRS [Fig. 1(c)] for all participants. Comparing fcNIRS and task-based NIRS approaches, very good concordance (DSC $\geq 0.80)^{61}$ was found for language cortical mapping among all participants (mean DSC $=0.81 \pm 0.13$ ) as well as for each age group: early childhood: mean $\mathrm{DSC}=0.73 \pm 0.12$, late childhood: mean DSC $=0.81 \pm 0.15$, adolescence: mean $\mathrm{DSC}=0.83 \pm 0.12$, and early adulthood: mean $\mathrm{DSC}=$ $0.84 \pm 0.13$. The one-way ANOVA results showed no statistical differences between groups for DSC $(p=0.405)$. Overall, these results show that fcNIRS and task-based NIRS provide similar language mapping for all age groups from age 3 to early adulthood.

Individual results, shown in Table 2, revealed that $85 \%$ (28/33) of all participants showed good concordance (DSC $\geq 0.70$ ) between both fcNIRS and task-based NIRS language mapping. Figure 3 shows individual results from one
Table 2 Comparison of language mapping and dominance using resting-state functional connectivity and task-based approaches. DSC values in bold represent participants with poor concordance (DSC <0.70). $\mathbf{N}$ indicates the non concordance between laterality indices from both approaches (fcNIRS and task-based NIRS).

\begin{tabular}{|c|c|c|c|c|}
\hline Participant & DSC & rs-fc LIs & task-based Lls & LI concordance \\
\hline 1 & 0.89 & $0.18(\mathrm{~L})$ & $0.98(\mathrm{~L})$ & $\mathrm{Y}$ \\
\hline 2 & 0.77 & $0.23(\mathrm{~L})$ & $0.27(\mathrm{~L})$ & $\mathrm{Y}$ \\
\hline 3 & 0.78 & 0.08 (B) & $0.17(\mathrm{~L})$ & $\mathbf{N}$ \\
\hline 4 & 0.70 & $0.12(\mathrm{~L})$ & $0.90(\mathrm{~L})$ & $\mathrm{Y}$ \\
\hline 5 & 0.54 & $0.29(\mathrm{~L})$ & $0.41(\mathrm{~L})$ & $\mathrm{Y}$ \\
\hline 6 & 0.71 & $0.15(\mathrm{~L})$ & $0.20(\mathrm{~L})$ & $\mathrm{Y}$ \\
\hline 7 & 0.96 & 0.37 (L) & $0.92(\mathrm{~L})$ & $\mathrm{Y}$ \\
\hline 8 & 0.94 & $0.11(\mathrm{~L})$ & $0.96(\mathrm{~L})$ & $\mathrm{Y}$ \\
\hline 9 & 0.69 & $0.11(\mathrm{~L})$ & $-0.13(\mathrm{R})$ & $\mathbf{N}$ \\
\hline 10 & 0.87 & $0.32(\mathrm{~L})$ & $0.39(\mathrm{~L})$ & $\mathrm{Y}$ \\
\hline 11 & 1 & $0.18(\mathrm{~L})$ & $0.53(\mathrm{~L})$ & $\mathrm{Y}$ \\
\hline 12 & 0.76 & 0.05 (B) & $0.10(\mathrm{~L})$ & $\mathbf{N}$ \\
\hline 13 & 0.64 & $0.24(\mathrm{~L})$ & $0.30(\mathrm{~L})$ & $\mathrm{Y}$ \\
\hline 14 & 0.62 & $0.31(\mathrm{~L})$ & $0.27(\mathrm{~L})$ & $\mathrm{Y}$ \\
\hline 15 & 0.97 & $0.06(\mathrm{~B})$ & $0.10(\mathrm{~L})$ & $\mathbf{N}$ \\
\hline 16 & 0.76 & $0.14(\mathrm{~L})$ & $0.13(\mathrm{~L})$ & $\mathrm{Y}$ \\
\hline 17 & 0.72 & $0.08(\mathrm{~B})$ & $0.08(\mathrm{~B})$ & $\mathrm{Y}$ \\
\hline 18 & 1 & $0.22(\mathrm{~L})$ & $-0.25(R)$ & $\mathbf{N}$ \\
\hline 19 & 0.73 & $0.12(\mathrm{~L})$ & $0.26(\mathrm{~L})$ & $\mathrm{Y}$ \\
\hline 20 & 0.87 & $0.04(\mathrm{~B})$ & 0.08 (B) & $\mathrm{Y}$ \\
\hline 21 & 0.71 & $0.21(\mathrm{~L})$ & $0.32(\mathrm{~L})$ & $\mathrm{Y}$ \\
\hline 22 & 0.76 & $0.16(\mathrm{~L})$ & $0.62(\mathrm{~L})$ & $\mathrm{Y}$ \\
\hline 23 & 0.92 & $0.06(\mathrm{~B})$ & $-0.07(\mathrm{~B})$ & $\mathrm{Y}$ \\
\hline 24 & 1 & 0.08 (B) & $-0.10(R)$ & $\mathbf{N}$ \\
\hline 25 & 0.74 & $0.17(\mathrm{~L})$ & $0.23(\mathrm{~L})$ & $\mathrm{Y}$ \\
\hline 26 & 1 & $0.13(\mathrm{~L})$ & $0.14(\mathrm{~L})$ & $Y$ \\
\hline 27 & 0.74 & $0.27(\mathrm{~L})$ & $0.11(\mathrm{~L})$ & $Y$ \\
\hline 28 & 0.65 & 0.04 (B) & 0.07 (B) & $\mathrm{Y}$ \\
\hline 29 & 0.94 & $0.03(\mathrm{~B})$ & 0.08 (B) & $\mathrm{Y}$ \\
\hline 30 & 0.93 & $0.18(\mathrm{~L})$ & $0.18(L)$ & $Y$ \\
\hline 31 & 0.72 & $0.10(\mathrm{~L})$ & 0.07 (B) & $\mathbf{N}$ \\
\hline 32 & 0.78 & 0.08 (B) & $0.2(\mathrm{~L})$ & $\mathbf{N}$ \\
\hline 33 & 0.96 & $0.38(\mathrm{~L})$ & $0.54(\mathrm{~L})$ & $\mathrm{Y}$ \\
\hline
\end{tabular}


(a) fCNIRS

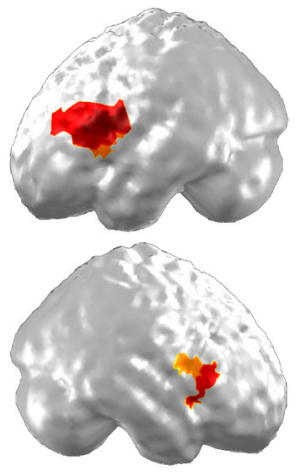

(c)
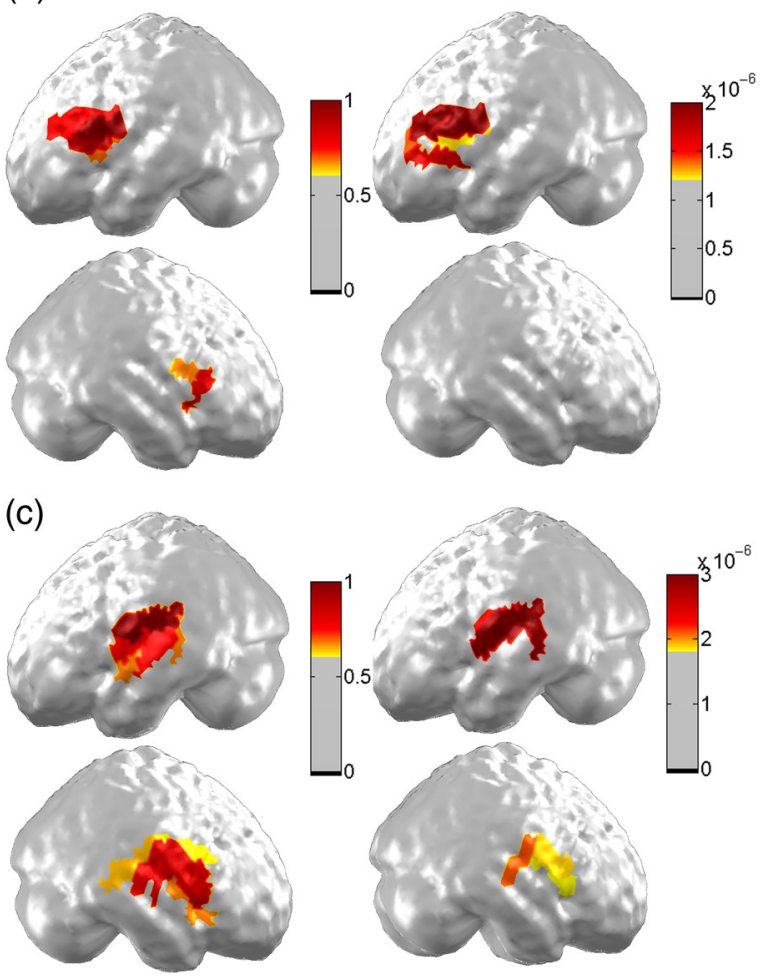

(b)

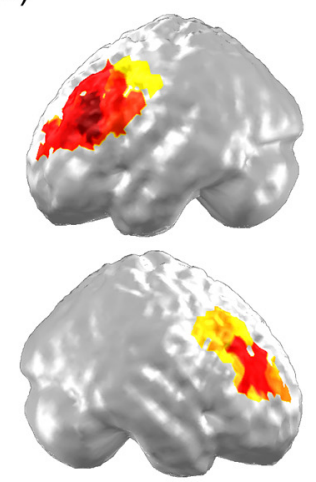

(d)

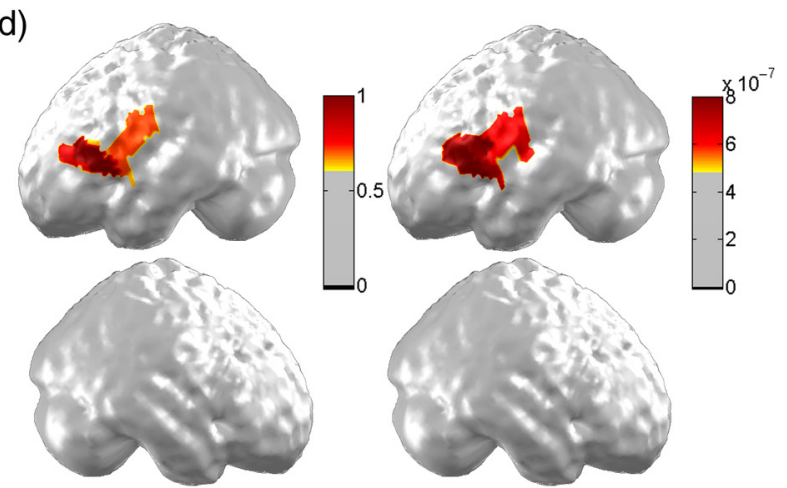

Task-based NIRS

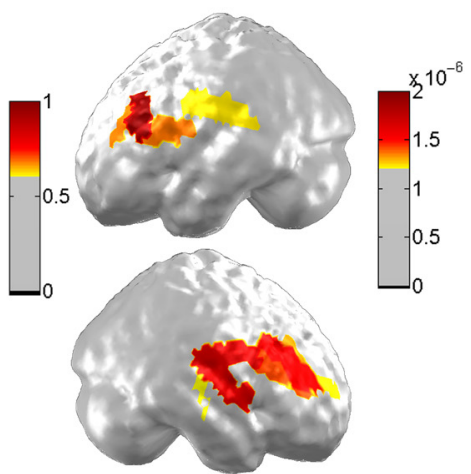

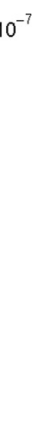

Fig. 3 Individual language mapping using fcNIRS and task-based NIRS (during a verbal fluency task) for each age group. (a) Cerebral localization of language function in a 3-year-old girl (participant 1 in the tables) using resting-state fcNIRS and task-based NIRS ( $\mathrm{HbO}$ concentration in Umolar) while the participant performed a verbal fluency task aloud. For both techniques, language brain networks were localized in typically expressive language-associated regions: left inferior frontal region or Broca's area. Good statistical concordance between fcNIRS and task-based NIRS maps was obtained in this participant $(\mathrm{DSC}=0.89$ ). (b) Language brain mapping in a 10-year-old boy (participant 12) reveals bilateral but left-greater-than-right, language functions. Good concordance $(\mathrm{DSC}=0.76)$ is found when comparing fcNIRS and task-based NIRS maps. (c) Very good concordance $(\mathrm{DSC}=0.97)$ is found between fcNIRS and task-based language mapping in a 11-year-old girl (participant 15). Although bilateral brain responses are found, both techniques revealed slightly left-greater-than-right hemispheric language activation in this participant. (d) Strong left hemisphere language dominance was found in a 30-year-old woman (participant 33) using both techniques, fcNIRS and task-based NIRS. Good spatial concordance was found for both mapping techniques (DSC $=0.96$ ).

participant in each age group and compares fcNIRS and taskbased NIRS language mapping.

\subsection{Language Laterality}

A comparison between laterality indices derived from fcNIRS and task-based NIRS for each participant revealed comparable hemispheric language dominance (kappa $=0.86, p<0.006$ ). Individual results (Table 2) show that for $76 \%(25 / 33)$ of all participants, there was agreement between the two approaches. More specifically, $83 \%(5 / 6)$ of early childhood participants, $75 \%(6 / 8)$ of late childhood participants, $73 \%(8 / 11)$ of adolescents, and $75 \%(6 / 8)$ of adults showed concordance between LIs calculated from fcNIRS (rs-fc LIs) and task-based NIRS (task-based LIs).

\section{Discussion and Conclusions}

Resting-state functional connectivity is a relatively modern method for evaluating regional interactions that occur in the brain when a subject is not performing an explicit task. Compelling studies have reported a highly correlated signal between left and right hemisphere cerebral regions comprised of motor, visual, auditory, and high-order cognitive systems, ${ }^{63-67}$ allowing for identification of functional networks. Resting-state functional connectivity can be investigated using various neuroimaging techniques including NIRS (fcNIRS). In the current study, we assessed the utility of fcNIRS for language localization in pediatric populations by comparing fcNIRS and task-based NIRS for language brain mapping and dominance in healthy participants.

For task-based NIRS, the language task was a child-friendly categorical verbal fluency task, which was used in many of our previous studies on expressive language localization in children, adolescents, and adults. ${ }^{25,48-50}$ Using this task, we showed similar expressive language lateralization patterns among children (ages 3 to 6 and 7 to 10), adolescents (ages 11 to 16), and young adults (ages 19 to 30). ${ }^{46}$ More specifically, strong left hemisphere responses along with weaker right hemisphere activation were found in all groups. Moreover, younger children (ages 3 to 6) showed smaller hemodynamic responses than adolescents and adults in both hemispheres, probably due to weaker performance by the younger children on the task. Hence, in the present 
study, we compared fcNIRS with a well-known language task-based paradigm to validate the reliability of the fcNIRS approach.

Overall, results show very good concordance between fcNIRS and task-based NIRS for language mapping in all age groups (early childhood, late childhood, adolescence, and early adulthood). In addition, strong agreement was found for language lateralization when comparing laterality indices derived from both techniques. A left hemisphere dominance was found in most participants, replicating the well-known left-lateralization phenomenon of language functions. ${ }^{68-70}$ Overall, these findings suggest that fcNIRS is a child-friendly approach that can be applied in individuals of various ages, and as young as age 3, to localize and lateralize language functions.

Although concordance between fcNIRS and task-based results for language lateralization was found in most participants $(25 / 33)$, inconsistencies were obtained in eight participants. We believe that the relatively short duration of each resting-state block (25 s) may account for these discrepancies. Although it may be difficult to apply with the pediatric population, the concordance ratio among language laterality indices may be increased by extending the resting recording duration from 5 to 10 consecutive minutes, as in previous studies. ${ }^{35,37,39}$

Another limitation of this study is potential contamination of the fcNIRS data by the language task activation. Although multiple measures were taken to prevent contamination (specific instructions given to all participants, fcNIRS data selected in the first rest period preceding category noun presentations for each block, a break of 2 to 3 min between blocks), the participants may still have been rehearsing words from the previous category or the residual $\mathrm{HbO}$ changes from the previous language task may have still been present. As in previous studies (e.g., Ref. 37), recording all fcNIRS data prior to task-based NIRS data would ensure that functional connectivity is measured without any potential contamination from the language task.

With appropriate NIRS coverage, fcNIRS could be used not only for language mapping but also to investigate other brain networks, such as motor and sensory functions, as well as default mode or executive control networks. This would be possible using NIRS data acquired with only a few minutes of rest in various pediatric populations. Although fcNIRS constitutes a useful and promising tool in clinical populations, including young children and patients with cognitive and behavioral impairments, further research is needed to validate its clinical applications, such as presurgical investigation of language functions in patients with brain tumor or intractable epilepsy.

\section{Disclosures}

No conflicts of interest, competing or financial interests, or otherwise, are declared by the 3 authors.

\section{Acknowledgments}

We are grateful to the study participants and their families. We also thank Dr. Pierre Bellec for providing valuable advice on resting-state functional connectivity analyses. This work was funded by the National Science and Engineering Research Council of Canada (NSERC, \#2015-04199), the SickKids Foundation (\#NI16-058), and the Fonds de Recherche du Québec Santé (FRQS, \#28811).

\section{References}

1. A. Gallagher, R. Béland, and M. Lassonde, "Techniques for presurgical exploration of language lateralization in children," in Advances in Medicine and Biology, L. V. Berhardt, Ed., pp. 87-110, Nova Science Publishers, Hauppauge (2010).

2. C. C. Tebo et al., "Evolution of cranial epilepsy surgery complication rates: a 32-year systematic review and meta-analysis," J. Neurosurg. 120(6), 1415-1427 (2014).

3. T. Loddenkemper, H. H. Morris, and G. Moddel, "Complications during the Wada test," Epilepsy Behav. 13(3), 551-553 (2008).

4. A. Haag et al., "The Wada test in Austrian, Dutch, German, and Swiss epilepsy centers from 2000 to 2005: a review of 1421 procedures," Epilepsy Behav. 13(1), 83-89 (2008).

5. C. A. Schevon et al., "Pediatric language mapping: sensitivity of neurostimulation and Wada testing in epilepsy surgery," Epilepsia 48(3), 539545 (2007).

6. S. G. Ojemann et al., "Localization of language function in children: results of electrical stimulation mapping," J. Neurosurg. 98(3), 465-470 (2003).

7. H. Urbach et al., "Presurgical MR imaging in epilepsy," Clin. Neuroradiol. 25(Suppl. 2), 151-155 (2015).

8. R. O. Suarez et al., "Passive fMRI mapping of language function for pediatric epilepsy surgical planning: validation using Wada, ECS, and FMAER," Epilepsy Res. 108(10), 1874-1888 (2014).

9. W. D. Gaillard et al., "fMRI language task panel improves determination of language dominance," Neurology 63, 1403-1408 (2004).

10. L. M. Balsamo, B. Xu, and W. D. Gaillard, "Language lateralization and the role of the fusiform gyrus in semantic processing in young children," Neuroimage 31(3), 1306-1314 (2006).

11. L. Hertz-Pannier et al., "Noninvasive assessment of language dominance in children and adolescents with functional MRI: a preliminary study," Neurology 48(4), 1003-1012 (1997).

12. J. I. Breier and A. C. Papanicolaou, "Spatiotemporal patterns of brain activation during an action naming task using magnetoencephalography," J. Clin. Neurophysiol. 25(1), 7-12 (2008).

13. W. S. Merrifield et al., "Hemispheric language dominance in magnetoencephalography: sensitivity, specificity, and data reduction techniques," Epilepsy Behav. 10(1), 120-128 (2007).

14. A. E. Fisher et al., "Interhemispheric differences of spectral power in expressive language: a MEG study with clinical applications," Int. J. Psychophysiol. 68(2), 111-122 (2008).

15. A. C. Papanicolaou et al., "Magnetoencephalographic mapping of the language-specific cortex," J. Neurosurg. 90(1), 85-93 (1999).

16. P. G. Simos et al., "Assessment of functional cerebral laterality for language using magnetoencephalography," J. Clin. Neurophysiol. 15(4), 364-372 (1998).

17. R. Salmelin et al., "Dynamics of brain activation during picture naming," Nature 368(6470), 463-465 (1994).

18. D. Lee et al., "Reliability of language mapping with magnetic source imaging in epilepsy surgery candidates," Epilepsy Behav. 8(4), 742749 (2006).

19. S. M. Bowyer et al., "MEG localization of language-specific cortex utilizing MR FOCUSS," Neurology 62, 2247-2255 (2004).

20. S. M. Bowyer et al., "Language laterality determined by MEG mapping with MR-FOCUSS," Epilepsy Behav. 6(2), 235-241 (2005).

21. P. R. Bauer et al., "Can fMRI safely replace the Wada test for preoperative assessment of language lateralisation? A meta-analysis and systematic review," J. Neurol. Neurosurg. Psychiatry 85(5), 581-588 (2014).

22. B. Abou-Khalil, "An update on determination of language dominance in screening for epilepsy surgery: the Wada test and newer noninvasive alternatives," Epilepsia 48(3), 442-455 (2007).

23. A. C. Papanicolaou et al., "Magnetocephalography: a noninvasive alternative to the Wada procedure," J. Neurosurg. 100(5), 867-876 (2004).

24. A. Villringer et al., "Near infrared spectroscopy (NIRS): a new tool to study hemodynamic changes during activation of brain function in human adults," Neurosci. Lett. 154(1-2), 101-104 (1993).

25. A. Gallagher et al., "Near-infrared spectroscopy as an alternative to the Wada test for language mapping in children, adults and special populations," Epileptic Disord. 9(3), 241-255 (2007).

26. E. Watanabe et al., "Non-invasive assessment of language dominance with near-infrared spectroscopic mapping," Neurosci. Lett. 256, 4952 (1998). 
27. P. Pouliot et al., "Nonlinear hemodynamic responses in human epilepsy: a multimodal analysis with fNIRS-EEG and fMRI-EEG," J. Neurosci. Methods 204(2), 326-340 (2012).

28. K. Wagner et al., "Who needs a Wada test? Present clinical indications for amobarbital procedures," J. Neurol. Neurosurg. Psychiatry 83(5), 503-509 (2012).

29. A. Gallagher, "The utility of near infrared spectroscopy in pediatric epilepsy," J. Pediatr. Epilepsy 2, 87-92 (2013).

30. A. T. Berg et al., "Frequency, prognosis and surgical treatment of structural abnormalities seen with magnetic resonance imaging in childhood epilepsy," Brain 132(Pt. 10), 2785-2797 (2009).

31. H. Liu et al., "Evidence from intrinsic activity that asymmetry of the human brain is controlled by multiple factors," Proc. Natl. Acad. Sci. U. S. A. 106(48), 20499-20503 (2009).

32. B. Biswal et al., "Functional connectivity in the motor cortex of resting human brain using echo-planar MRI," Magn. Reson. Med. 34, 537-541 (1995).

33. N. Tanaka and S. M. Stufflebeam, "Presurgical mapping of the language network using resting-state functional connectivity," Top. Magn. Reson. Imaging 25(1), 19-24 (2016).

34. U. Chaudhary et al., "Frontal activation and connectivity using nearinfrared spectroscopy: verbal fluency language study," Brain Res. Bull. 84(3), 197-205 (2011).

35. J. Li and L. Qiu, "Temporal correlation of spontaneous hemodynamic activity in language areas measured with functional near-infrared spectroscopy," Biomed. Opt. Express 5(2), 587-595 (2014).

36. B. Molavi et al., "Analyzing the resting state functional connectivity in the human language system using near infrared spectroscopy," Front. Hum. Neurosci. 7, 921 (2014).

37. Y. J. Zhang et al., "Detecting resting-state functional connectivity in the language system using functional near-infrared spectroscopy," J. Biomed. Opt. 15(4), 047003 (2010).

38. R. C. Mesquita, M. A. Franceschini, and D. A. Boas, "Resting state functional connectivity of the whole head with near infrared spectroscopy," Biomed. Opt. Express 1(1), 324-336 (2010).

39. S. Sasai et al., "A NIRS-fMRI study of resting state network," Neuroimage 63(1), 179-193 (2012).

40. H. Niu et al., "Test-retest reliability of graph metrics in functional brain networks: a resting-state fNIRS study," PLoS One 8(9), e72425 (2013).

41. G. Gratton and M. Fabiani, "Optical imaging of brain function," in Neuroergonomics: The Brain at Work, R. P. M. Mizzo, Ed., Oxford University Press, Cambridge (2007).

42. B. R. White et al., "Resting-state functional connectivity in the human brain revealed with diffuse optical tomography," Neuroimage 47(1), 148-156 (2009).

43. H. Obrig et al., "Spontaneous low frequency oscillations of cerebral hemodynamics and metabolism in human adults," Neuroimage 12(6), 623-639 (2000)

44. C. M. Lu et al., "Use of fNIRS to assess resting state functional connectivity," J. Neurosci. Methods 186(2), 242-249 (2010).

45. D. V. Bishop, M. J. Hardiman, and J. G. Barry, "Is auditory discrimination mature by middle childhood? A study using time-frequency analysis of mismatch responses from 7 years to adulthood," Dev. Sci. 14(2), 402-416 (2011).

46. N. Paquette et al., "Developmental patterns of expressive language hemispheric lateralization in children, adolescents and adults using functional near-infrared spectroscopy," Neuropsychologia 68, 117125 (2015).

47. C. J. Holmes et al., "Enhancement of MR images using registration for signal averaging," J. Comput. Assist. Tomogr. 22(2), 324-333 (1998).

48. P. Vannasing et al., "Potential brain language reorganization in a boy with refractory epilepsy; an fNIRS-EEG and fMRI comparison," Epilepsy Behav. Case Rep. 5, 34-37 (2016).

49. A. Gallagher et al., "A noninvasive, presurgical expressive and receptive language investigation in a 9-year-old epileptic boy using near-infrared spectroscopy," Epilepsy Behav. 12(2), 340-346 (2008).

50. A. Gallagher et al., "Non-invasive pre-surgical investigation of a 10 year-old epileptic boy using simultaneous EEG-NIRS," Seizure 17(6), 576-582 (2008).

51. R. C. Oldfield, "The assessment and analysis of handedness: the Edinburgh inventory," Neuropsychologia 9(1), 97-113 (1971).
52. G. Strangman, M. A. Franceschini, and D. A. Boas, "Factors affecting the accuracy of near-infrared spectroscopy concentration calculations for focal changes in oxygenation parameters," Neuroimage 18(4), 865-879 (2003).

53. A. Duncan et al., "Measurement of cranial optical path length as a function of age using phase resolved near infrared spectroscopy," Pediatr. Res. 39(5), 889-894 (1996).

54. J. H. Meek et al., "Regional changes in cerebral haemodynamics as a result of a visual stimulus measured by near infrared spectroscopy," Proc. R. Soc. B: Biol. Sci. 261(1362), 351-356 (1995).

55. A. M. Siegel et al., "Temporal comparison of functional brain imaging with diffuse optical tomography and fMRI during rat forepaw stimulation," Phys. Med. Biol. 48(10), 1391-1403 (2003).

56. P. Bellec et al., "Multi-level bootstrap analysis of stable clusters in resting-state fMRI," Neurolmage 51(3), 1126-1139 (2010).

57. C. E. Sanchez, J. E. Richards, and C. R. Almli, "Age-specific MRI templates for pediatric neuroimaging," Dev. Neuropsychol. 37(5), 379-399 (2012).

58. C. E. Sanchez, J. E. Richards, and C. R. Almli, "Neurodevelopmental MRI brain templates for children from 2 weeks to 4 years of age," Dev. Psychobiol. 54(1), 77-91 (2012).

59. W. Yuan et al., "fMRI shows atypical language lateralization in pediatric epilepsy patients," Epilepsia 47(3), 593-600 (2006).

60. K. H. Zou et al., "Statistical validation of image segmentation quality based on a spatial overlap index," Acad. Radiol. 11(2), 178-189 (2004).

61. A. P. Zijdenbos et al., "Morphometric analysis of white matter lesions in MR images: method and validation," IEEE Trans. Med. Imaging 13(4), 716-724 (1994).

62. S. Sun, "Meta-analysis of Cohen's kappa," Health Serv. Outcomes Res. Method. 11(3), 145-163 (2011).

63. B. Biswal et al., "Hypercapnia reversibly suppresses low-frequency fluctuations in the human motor cortex during rest using echo-planar MRI," J. Cereb. Blood Flow Metab. 17(3), 301-308 (1997).

64. D. Cordes et al., "Mapping functionally related regions of brain with functional connectivity MR imaging," AJNR Am. J. Neuroradiol. 21(9), 1636-1644 (2000).

65. M. D. Fox and M. E. Raichle, "Spontaneous fluctuations in brain activity observed with functional magnetic resonance imaging," Nat. Rev. Neurosci. 8(9), 700-711 (2007).

66. M. D. Greicius et al., "Functional connectivity in the resting brain: a network analysis of the default mode hypothesis," Proc. Natl. Acad. Sci. U. S. A. 100(1), 253-258 (2003).

67. J. S. Damoiseaux et al., "Consistent resting-state networks across healthy subjects," Proc. Natl. Acad. Sci. U. S. A. 103(37), 1384813853 (2006).

68. P. Broca, "Remarques sur le siège de la faculté du langage articulé suivies d'une observation d'aphémie," Bull. Soc. Anatomique 36, 330-357 (1961).

69. J. Pujol et al., "Cerebral lateralization of language in normal left-handed people studied by functional MRI," Neurology 52(5), 1038 (1999).

70. J. A. Springer et al., "Language dominance in neurologically normal and epilepsy subjects: a functional MRI study," Brain 122(Pt. 11), 2033-2046 (1999).

Anne Gallagher is an assistant research professor in the Psychology Department, Université de Montréal, and director of the Laboratoire d'Imagerie Optique en Neurodéveloppement (LION Lab) at CHU Sainte-Justine, Montreal. She received her PhD in neuropsychology from the Université de Montréal and a postdoctoral research fellowship from Harvard Medical School. Her research program focuses on developing clinical near-infrared spectroscopy (NIRS) and electroencephalography (EEG) tools to improve neurodevelopmental and surgical outcomes in various pediatric populations.

Julie Tremblay is an electrical engineer at the LION Lab. She holds a BSc degree from the Université de Sherbrooke. Her work is dedicated to creating and developing adapted, flexible NIRS and EEG data acquisition, analyses, and visualization software.

Phetsamone Vannasing is a senior electrophysiology technician at the LION Lab. She has worked with healthy and sick children for over 20 years and has performed hundreds of NIRS and EEG recordings, data analyses, and interpretations. 